\title{
Ureteroscopy in Pregnant Women for Ureteral Stone
}

\author{
Marcelo Travassos, M.D., ${ }^{1}$ Isaac Amselem, M.D., ${ }^{2}$ Newton Sá Filho, M.D., ${ }^{2}$ Marshall Miguel, M.D., ${ }^{2}$ \\ Américo Sakai, M.D., ${ }^{3}$ Horácio Consolmagno, M.D., ${ }^{3}$ Marcos Nogueira, M.D., ${ }^{3}$ and Oscar Fugita, M.D. ${ }^{1,2,3}$
}

\begin{abstract}
Introduction: The occurrence of urolithiasis in pregnancy represents a challenge in both diagnosis and treatment of this condition, because it presents risks not only to the mother but also to the fetus. Surgical treatment may be indicated for patients with infection, persistent pain, and obstruction of a solitary kidney. We present our experience on the management of pregnant patients with ureteral calculi and a review of the literature.

Materials and Methods: The charts of 19 pregnant patients with obstructive ureteral calculi were retrospectively reviewed. Gestational age ranged from 13 to 33 weeks. In all patients, ureteral stone was diagnosed on abdominal ultrasound. In regard to localization, 15 calculi were in the distal ureter, 3 in the proximal ureter, and 1 in the interior of an ureterocele. Calculi size ranged from 6 to $10 \mathrm{~mm}$ (mean, $8 \mathrm{~mm}$ ). The following criteria were used to indicate ureteroscopy: persistent pain with no improvement after clinical treatment, increase in renal dilation, or presence of uterine contractions. Nine patients $(47.3 \%)$ were submitted to ureteroscopy. All calculi $(100 \%)$ were removed with a stone basket extractor under continuous endoscopic vision. None of the calculi demanded the use of a lithotriptor.

Results: Nine patients (47.3\%) treated with clinical measurements presented no obstetric complications and spontaneous elimination of the calculi. Nine patients (47.3\%) submitted to ureteroscopy had no surgical complications. There was remission of pain in all cases after ureteroscopy and ureteral catheter placement.

Conclusion: The diagnosis and treatment of ureteral lithiasis in pregnant women present potential risks for the fetus and the mother. Conservative management is the first option, but ureteroscopy may be performed with safety and high success rates.
\end{abstract}

\section{Introduction}

$\mathbf{U}$ ROLITHIASIS IN PREGNANCY is a rare situation $(0.07 \%)$. $^{1,2}$ Urolithiasis is diagnosed in the majority of patients only when calculi become symptomatic. ${ }^{3}$ The occurrence of urolithiasis in pregnancy represents a challenge in both diagnosis and treatment of this condition, because it presents risks not only to the mother but also to the fetus.

Pregnancy may mask the symptoms and signs of renal colic, and many patients present abdominal pain that is distinct from the typical renal colic. On the other hand, renal colic is the most common cause of hospitalization for abdominal pain of nonobstetric origin in gestation. ${ }^{4}$

Conservative measurements are indicated in the majority of cases, because $70 \%$ to $80 \%$ of patients will spontaneously eliminate the calculi. ${ }^{5}$ In cases of patients with infection, persistent pain, and obstruction of a solitary kidney, surgical treatment may be indicated.
Additionally, fetal risks related to exposure to X-rays, to medications, and to anesthetic agents may limit the use of diagnostic and therapeutic methods.

We present our experience on the management of pregnant patients with ureteral calculi and a review of the literature.

\section{Material and Methods}

The charts of 19 pregnant patients with obstructive ureteral calculi were retrospectively reviewed. Gestational age ranged from 13 to 33 weeks.

In all patients, ureteral stone was diagnosed on abdominal ultrasonography. In regard to localization, 15 calculi were in the distal ureter, 3 in the proximal ureter, and 1 in the interior of an ureterocele. Calculi size ranged from 6 to $10 \mathrm{~mm}$ (mean $8 \mathrm{~mm}$ ).

In all patients, conservative management was firstly indicated for at least 24 hours consisting on analgesia, hydration,

\footnotetext{
${ }^{1}$ Department of Urology, São Paulo State University, Botucatu, Brazil.

${ }^{2}$ Department of Urology, Hospital São Camilo Santana, São Paulo, Brazil.

${ }^{3}$ Department of Surgery, Hospital Universitário-USP, São Paulo, Brazil.
} 
and antibiotics administration, when there was associated urinary tract infection. The following criteria were used to indicate ureteroscopy: persistent pain with no improvement after clinical treatment, increase in renal dilation, or presence of uterine contractions.

Nine patients $(47.3 \%)$ were submitted to ureteroscopy due to persistent pain and hydronephrosis. The procedure was initiated with the passage of one guide wire introduced up to the kidney. Next, a semirigid ureteroscope was used for localization of the calculi and its removal with a stone basket extractor under continuous endoscopic vision. None of the calculi demanded the use of a lithotriptor. All patients had Double-J catheter left in place.

In one patient $(5.8 \%)$ who presented with fever and leukocyturia, the only procedure performed was the insertion of a Double-J catheter. No attempt was made to remove the stone due to the urinary tract infection. Fluoroscopy was not used in any of the cases.

The Institutional Review Board approved the clinical protocol, and the patients gave their informed consent.

\section{Results}

Nine patients $(47.3 \%)$ treated with clinical measurements presented no obstetric complications and spontaneous elimination of the calculi. One patient $(5.4 \%)$ had a Double-J stent passed up at 28 weeks gestational age. The ureteral catheter was left in place until delivery (at 39 weeks gestational age) when a ureteroscopy was performed to remove the stone and the stent.

Nine patients $(47.3 \%)$ submitted to ureteroscopy had no surgical complications. Mean gestational age at the moment of procedure was 19 weeks (13-28 weeks). There was remission of pain in all cases after ureteroscopy and ureteral catheter placement. The Double-J catheter was left in place for 7 days with no recurrence of stones and pain. Mean gestational age of delivery in these nine patients was 40 weeks (38-42 weeks). None of the indications for preterm delivery was caused by urological complications.

\section{Discussion}

Renal calculi represent one of the most common causes of abdominal pain during pregnancy, and, frequently, hospitalization may be required. ${ }^{4}$

It occurs most commonly in multiple pregnancy, ${ }^{6}$ particularly in the second and third trimesters of gestation. ${ }^{7}$ It is present with equal frequency in both sides, ${ }^{8}$ although some series demonstrate a predisposition for the right side compared to the left. ${ }^{9}$

Diagnosis of ureteral calculi in pregnancy may be hindered by physiological dilation of the upper urinary tract, which occurs in up to $90 \%$ of pregnancies, due to compression of the ureter by the uterus. ${ }^{10}$ Gestation does not promote calculi formation, and the most present symptom is flank pain that occurs in $84 \%$ to $100 \%$ of cases. ${ }^{6}$

Microscopic or macroscopic hematuria can be present. However, Stothers and Lee reported that microscopic hematuria is not evident in $25 \%$ of patients with diagnosed calculi. ${ }^{6}$ Parulkar et al diagnosed lithiasis in 40 of 66 pregnancies with hematuria ${ }^{11}$ and concluded that microscopic hematuria is not discriminatory for diagnosing calculi, and despite being a frequent sign, it is not specific. ${ }^{11}$
The diagnosis may be difficult because of the limitation on the use of imaging exams. Ultrasonography and plain X-rays of the abdomen are the main exams in the evaluation of renal colic in pregnant women. Physiological dilation of the pyelocaliceal system during pregnancy, particularly in the right side, diminishes substantially the sensitivity of ultrasonography. Ultrasound sensitivity for the diagnosis of urinary lithiasis varies between $34 \%{ }^{12}$ and $98 \%{ }^{6}$ Parulkar et al demonstrated a sensitivity of $95.2 \%{ }^{11}$ for diagnosis of middle ureter lithiasis.

When ultrasonography is not conclusive, radiographic evaluation may be indicated. Swartz ${ }^{13}$ identified the first trimester as the period of greatest risk for fetal malformation and spontaneous abortion after exposure to radiation. The author reported that exposure to 25 to $80 \mathrm{rad}$ doubles the teratogenic risk in the fetus. Radiation exposure after a plain X-ray is of about $20 \mathrm{mrad}$, less than $1 \%$ of the critical dose. Intravenous pyelography with just a nephrogram at 30 seconds and a film at 20 minutes may be performed and presents minimal risk to the fetus in the second and third trimesters of pregnancy. ${ }^{13}$ Fluoroscopy must be avoided.

Computerized tomography of the abdomen and pelvis is not a diagnostic option. The radiation exposure is of about $2.3 \mathrm{cGy}$, and it increases by 2.4 times the risks of cancer in children. ${ }^{14}$ Magnetic resonance imaging of the urinary tract can identify the site of obstruction, but it is not effective in identifying the calculi. Further, it is an expensive method of little availability that offers diagnosis based on indirect signs. ${ }^{15}$ In all patients of our series, diagnosis was based on ultrasonography findings.

Approximately $66 \%$ to $85 \%$ of pregnant patients with renal colic present spontaneous elimination of calculi when treated conservatively with hydration and analgesics. ${ }^{6,16}$ Parulkar refers a rate of spontaneous elimination of calculi of $64.3 \%$, in 70 pregnant women. The author recommends surgical intervention only if the patient does not eliminate the calculi within 4 weeks of parturition or in the presence of intense pain, infection, or anuria in solitary kidney. ${ }^{11}$ In our series, 9 out of 17 patients $(52.9 \%)$ eliminated the calculi spontaneously.

When conservative management is not successful, implantation of ureteral prosthesis, percutaneous nephrostomy, or ureteroscopy is the surgical option.

The Double-J stents must be located with a minimum of radioscopy exposition or, even better, with ultrasonography guidance. Pregnant patients with urinary tract infection and concomitant lithiasis are better treated with the passage of a Double-J catheter. They must not be submitted to ureteroscopy to avoid dissemination of the infection. Encrustation of catheters may demand frequent replacements in these patients. Kavoussi et al suggest that catheters must be changed every 6 to 8 weeks. ${ }^{17}$ Occasionally, the catheters may migrate to the bladder due to physiological dilation. ${ }^{6}$ Implantation of catheters should be performed preferentially at the end of the second or third trimester.

Some patients submitted to a Double-J catheter implantation may present continuous urinary symptoms and pain in the flank due to an ureteral vesical reflux. In these cases, percutaneous nephrostomy may be proposed as an alternative therapy. ${ }^{18}$ However, the good results of fragmentation and calculi removal during pregnancy in the last decade diminished the indications of placement of ureteral catheters and nephrostomy tubes. ${ }^{19}$ 
Extracorporeal shock wave lithotripsy is not indicated during gestation due to the risks of placental displacement, miscarriage, and malformations. ${ }^{20}$

The ureteral dilation present during pregnancy may facilitate the insertion of ureteroscopes and instruments without the necessity of dilatation of the ureteral meatus. Frequently, the physiological meatus dilation allows the extraction of the calculi without fragmentation, only with the use of a basket stone extractor.

Watterson et al demonstrated a free calculi rate of $89 \%$ in patients submitted to ureterolithotripsy with the Holmium laser. The authors treated eight women with a mean calculi size of $8 \mathrm{~mm}$. The mean time of fluoroscopy use was 0.19 minutes (0-1.3 minutes), and in four cases, fluoroscopy was not used. ${ }^{20}$ The depth of thermal energy of the laser (between 0.5 and $1 \mathrm{~mm}$ ) causes little effect in periureteral tissue and lower intensity of sound compared to other sources such as ultrasound and electrohydraulics. ${ }^{20}$ Ulvik et al report that the use of ultrasound for calculi fragmentation may produce sound sufficiently loud to harm the hearing of the fetus. ${ }^{21}$ However, Karlsen et al in an in vitro study comparing different intraluminal lithotripters concluded that the sound emitted by an endoscopic lithotriptor in the ureter during pregnancy is unlikely to be harmful to the fetal hearing. ${ }^{22}$

The risk of major complications to the mother and fetus for urologists who have large experience with ureteroscopic procedures should be less than $2 \% .{ }^{23}$ In our experience, ureteroscopy for ureteral stones during pregnancy is a safe and reliable procedure. However, longer follow-up and more patients are needed to get definitive conclusions.

\section{Conclusion}

The diagnosis and treatment of ureteral lithiasis in pregnant women present potential risks for the fetus and the mother.

Conservative management is the first choice, but ureteroscopy may be performed with safety and high success rates.

\section{Disclosure Statement}

No competing financial interests exist.

\section{References}

1. Meria P, Anidjar M, Hermieu JF, Boccon-Gibod L. Urinary lithiasis and pregnancy. Prog Urol 1993;3:937-943.

2. Rodriguez PN, Klein AS. Management of urolithiasis during pregnancy. Surg Gynecol Obstet 1988;166:103-106.

3. Maikranz P, Lindheimer M, Coe F. Nephrolithiasis in pregnancy. Baillieres Clin Obstet Gynaecol 1994;8:375-386.

4. Folger GK. Pain and pregnancy; treatment of painful states complicating pregnancy, with particular emphasis on urinary calculi. Obstet Gynecol 1955;5:513-518.

5. Evans HJ, Wollin TA. The management of urinary calculi in pregnancy. Curr Opin Urol 2001;11:379-384.

6. Stothers L, Lee LM. Renal colic in pregnancy. J Urol 1992; 148:1383-1387.

7. Denstedt JD, Razvi H. Management of urinary calculi during pregnancy. J Urol 1992;148:1072-1074.
8. Lattanzi DR, Cook WA. Urinary calculi in pregnancy. Obstet Gynecol 1980;56:462-466.

9. Cumming DC, Taylor PJ. Urologic and obstetric significance of urinary calculi in pregnancy. Obstet Gynecol 1979;53:505508.

10. Swanson SK, Heilman RL, Eversman WG. Urinary tract stones in pregnancy. Surg Clin North Am 1995;75:123142.

11. Parulkar BG, Hopkins TB, Wollin MR, Howard PJ Jr., Lal A. Renal colic during pregnancy: A case for conservative treatment. J Urol 1998;159:365-368.

12. Ellenbogen PH, Scheible FW, Talner LB, Leopold GR. Sensitivity of gray scale ultrasound in detecting urinary tract obstruction. AJR Am J Roentgenol 1978;130:731-733.

13. Swartz HM, Reichling BA. Hazards of radiation exposure for pregnant women. JAMA 1978;239:1907-1908.

14. McAleer SJ, Loughlin KR. Nephrolithiasis and pregnancy. Curr Opin Urol 2004;14:123-127.

15. Spencer JA, Tomlinson AJ, Weston MJ, Lloyd SN. Early report: Comparison of breath-hold MR excretory urography, Doppler ultrasound and isotope renography in evaluation of symptomatic hydronephrosis in pregnancy. Clin Radiol 2000;55:446-453.

16. Jones WA, Correa RJ Jr., Ansell JS. Urolithiasis associated with pregnancy. J Urol 1979;122:333-335.

17. Kavoussi LR, Albala DM, Basler JW, Apte S, Clayman RV. Percutaneous management of urolithiasis during pregnancy. J Urol 1992;148(3 Pt 2):1069-1071.

18. Menon M, Resnick MI. Urinary lithiasis: Etiology, diagnosis, and medical management. In: Walsh PC, Retik AB, Wein AJ, Vaughan ED, eds. Campbell's Urology. 8th ed. Philadelphia: Elsevier Science, 2002, pp. 3229-3292.

19. Watterson JD, Girvan AR, Beiko DT, Nott L, Wollin TA, Razvi H, Denstedt JD. Ureteroscopy and holmium: YAG laser lithotripsy: An emerging definitive management strategy for symptomatic ureteral calculi in pregnancy. Urology 2002;60:383-387.

20. Chaussy CG, Fuchs GJ. Current state and future developments of noninvasive treatment of human urinary stones with extracorporeal shock wave lithotripsy. J Urol 1989; 141(3 Pt 2):782-789.

21. Ulvik NM, Bakke A, Høisaeter PA. Ureteroscopy in pregnancy. J Urol 1995;154:1660-1663.

22. Karlsen SJ, Bull-Njaa T, Krokstad A. Measurement of sound emission by endoscopic lithotripters: An in vitro study and theoretical estimation of risk of hearing loss in a fetus. J Endourol 2001;15:821-826.

23. Evans HJ, Wollin TA. The management of urinary calculi in pregnancy. Curr Opin Urol 2001;11:379-384.

Address reprint requests to: Oscar Fugita, M.D. Department of Urology Faculdade De Medicina De Botucatu-UNESP Av. Indianápolis, 2244 04062-002 São Paulo Brazil

E-mail: fugita@fmb.unesp.br 
